\title{
Minor mergers and the size evolution of elliptical galaxies
}

\author{
Thorsten Naab ${ }^{1}$, Peter H. Johansson ${ }^{1}$, Jeremiah P. Ostriker ${ }^{2}$ \\ 1 Universitäts-Sternwarte München, Scheinerstr. 1, D-81679 München, Germany; ${ }^{2}$ \\ Department of Astrophysics, Peyton Hall, Princeton, USAnaab@usm. Imu. de
}

\begin{abstract}
Using a high resolution hydrodynamical cosmological simulation of the formation of a massive spheroidal galaxy we show that elliptical galaxies can be very compact and massive at high redshift in agreement with recent observations. Accretion of stripped in-falling stellar material increases the size of the system with time and the central concentration is reduced by dynamical friction of the surviving stellar cores. In a specific case of a spheroidal galaxy with a final stellar mass of $1.5 \times 10^{11} M_{\odot}$ we find that the effective radius $r_{e}$ increases from $0.7 \pm 0.2 \mathrm{kpc}$ at $\mathrm{z}=3$ to $r_{e}=2.4 \pm 0.4 \mathrm{kpc}$ at $\mathrm{z}=0$ with a concomitant decrease in the effective density of an order of magnitude and a decrease of the central velocity dispersion by approximately $20 \%$ over this time interval. A simple argument based on the virial theorem shows that during the accretion of weakly bound material (minor mergers) the radius can increase as the square of the mass in contrast to the usual linear rate of increase for major mergers. By undergoing minor mergers compact high redshift spheroids can evolve into present-day systems with sizes and concentrations similar to observed local ellipticals. This indicates that minor mergers may be the main driver for the late evolution of sizes and densities of early-type galaxies.
\end{abstract}

Subject headings: galaxies: elliptical - galaxies: interaction- galaxies: structure - galaxies: evolution - methods: numerical

\section{Introduction}

There is recent observational evidence that a significant fraction of massive evolved spheroidal stellar systems is already in place at redshift $z \geq 2$. However, only a small percentage of these galaxies is fully assembled (van Dokkum et al. 2008). The galaxies are smaller by a factor of three to five compared to present-day ellipticals at similar masses (Daddi et al. 2005; Longhetti et al. 2007; Toft et al. 2007; Trujillo et al. 2007; van Dokkum et al. 2008; 
Cimatti et al. 2008; Saracco et al. 2008). Their effective stellar mass densities are at least one order of magnitude higher (van Dokkum et al. 2008; Damjanov et al. 2009) with significantly higher surface brightnesses compared to their low redshift analogs (Cimatti et al. 2008).

These observations are difficult to reconcile with some current idealized formation scenarios for elliptical galaxies. A simple conclusion from the data is that most early-type galaxies can neither have fully formed in a simple monolithic collapse nor a binary merger of gas-rich disks at high redshift, unless their increase in size can be explained by secular processes such as adiabatic expansion driven by stellar mass loss and/or strong feedback (Damjanov et al. 2009; Fan et al. 2008). Additionally, simple passive evolution of the stellar population is in contradiction with observations of local ellipticals (van Dokkum et al. 2008).

Dry (i.e. gas-poor, collisionless) mergers and stellar accretion events are the prime candidates for the strong mass and size evolution of stellar spheroids at $z<2$ (Naab et al. 2006; Khochfar \& Silk 2006; Bell et al. 2006a, bi ; Ruszkowski \& Springel 2009; Hopkins et al. 2009; van der Wel et al. 2009) as the additional presence of a dissipative component in a major merger event would limit the size increase (see e.g. Ciotti et al. 2007). The observed ellipticals are already very massive at high redshift, thus we expect from the shape of the mass function that minor mergers should be much more common than major mergers until $\mathrm{z}=0$.

Massive early-type galaxies may undergo not more than one major merger (with typically low cool gas content, see also Whitaker \& van Dokkum 2008) since $z=0.7$ (Bell et al. 2006a, see also McIntosh et al. 2008) with a significant contribution from minor mergers for the mass buildup (Bundy et al. 2009). The low number of observed major early-type mergers is also supported by theoretical evidence that massive $\left(\approx 10^{11-12} M_{\odot}\right)$ halos at $z=2$ typically experience only one major merger or less until $z=0$ and minor mergers are much more common (Genel et al. 2008b; Khochfar \& Silk 2008). On average, this is not enough to account for the required mass and size growth (see also Damjanov et al. 2009) as major dry mergers at most increase the size of a simple one component system by a factor of two and allowing for dark matter halos reduces the size growth further (Nipoti et al. 2003; Naab \& Trujillo 2006; Hopkins et al. 2008).

In this Letter we use, as a proof of principle, a very high resolution cosmological simulation of the formation of a spheroid with no major mergers below $z=3$ in combination with simple scaling relations to show that the observed rapid size growth and density evolution of spheroidal galaxies can be explained by minor mergers and small accretion events. The problem is computationally very expensive. At high redshift the observed ellipticals have 
half-mass sizes of $<1 \mathrm{kpc}$ with accreting subsystems of even smaller size. As we know from isolated merger simulations (see e.g. Naab \& Burkert 2003), to resolve such a system reasonably well we require a force softening of $10 \%$ of the effective radius, which in our case is of the order of $100 \mathrm{pc}$ and we require particle numbers of $\approx 10^{7}$ to simulate the galaxy in a full cosmological context over a Hubble time. Finally, to accurately follow the kinematics high force and integration accuracy are required.

\section{Size evolution and minor mergers}

Using the virial theorem we make a simple estimate of how an initial one-component stellar systems evolves when mass in stellar systems is added. We assume that a compact initial stellar system has formed dissipatively from stars. This system has a total energy $E_{i}$, a mass $M_{i}$, a gravitational radius $r_{a, i}$, and the mean square speed of the stars is $\left\langle v_{i}^{2}\right\rangle$. According to the virial theorem (Binney \& Tremaine 2008) the total energy of the system is

$$
\begin{aligned}
E_{i} & =K_{i}+W_{i}=-K_{i}=\frac{1}{2} W_{i} \\
& =-\frac{1}{2} M_{i}\left\langle v_{i}^{2}\right\rangle=-\frac{1}{2} \frac{G M_{i}^{2}}{r_{g, i}} .
\end{aligned}
$$

We then assume that systems are accreted with energies totaling $E_{a}$, masses totaling $M_{a}$, gravitational radii $r_{a, i}$ and mean square speeds averaging $\left\langle v_{a}^{2}\right\rangle$. We define the fractional mass increase from all the accreted material $\eta=M_{a} / M_{i}$ and the total kinetic energy of the material as $K_{a}=(1 / 2) M_{a}\left\langle v_{a}^{2}\right\rangle$, further defining $\epsilon=\left\langle v_{a}^{2}\right\rangle /\left\langle v_{i}^{2}\right\rangle$. Assuming energy conservation (orbital parameters from cosmological simulations (Khochfar \& Burkert 2006) indicate that most halos merge on parabolic orbits), the total energy of the final system is

$$
\begin{aligned}
E_{f} & =E_{i}+E_{a}=-\frac{1}{2} M_{i}\left\langle v_{i}^{2}\right\rangle-\frac{1}{2} M_{a}\left\langle v_{a}^{2}\right\rangle \\
& =-\frac{1}{2} M_{i}\left\langle v_{i}^{2}\right\rangle-\frac{1}{2} \eta M_{i} \epsilon\left\langle v_{i}^{2}\right\rangle \\
& =-\frac{1}{2} M_{i}\left\langle v_{i}^{2}\right\rangle(1+\epsilon \eta) \\
& =-\frac{1}{2} M_{f}\left\langle v_{f}^{2}\right\rangle .
\end{aligned}
$$

The mass of the final system is $M_{f}=M_{i}+M_{a}=(1+\eta) M_{i}$. Therefore the ratio of the final to initial mean square speeds is 


$$
\frac{\left\langle v_{f}^{2}\right\rangle}{\left\langle v_{i}^{2}\right\rangle}=\frac{(1+\eta \epsilon)}{1+\eta} .
$$

Similarly, the ratio of the final to initial gravitational radius is

$$
\frac{r_{g, f}}{r_{g, i}}=\frac{(1+\eta)^{2}}{(1+\eta \epsilon)}
$$

and for the ratio of the densities we get

$$
\frac{\rho_{f}}{\rho_{i}}=\frac{(1+\eta \epsilon)^{3}}{(1+\eta)^{5}}
$$

If during one or more mergers the initial stellar system increases its mass by a factor of two then $\eta=1$. This mass increase can be caused by one equal-mass merger in which case the mean square velocities of the two systems are identical and remain unchanged in the final system (Eqn. 3). The radius increases by a factor of two (Eqn. 4) and the density drops by a factor of four (Eqn. 5) (see also Hopkins et al. 2009). If, however, the total mass increase by a factor of two is caused by accretion of very small systems with $\left\langle v_{a}^{2}\right\rangle<<\left\langle v_{i}^{2}\right\rangle$ or $\epsilon<<1$, then the mean square velocities are reduced by a factor two, the radius is four times larger and the density is reduced by a factor of 32 with respect to the initial system (see also Bezanson et al. (2009) for a similar derivation of the scaling relations). We know from the shape of the Schechter function for the distribution of stellar masses that a massive system $\left(m>M_{*}\right)$ accretes most of its mass from lower mass systems and thus the simple calculation above makes it very plausible that even though major mergers do occur minor mergers are the main driver for the evolution in size and density of massive galaxies.

\section{High resolution simulations of an individual galaxy halo}

We have performed a cosmological N-body/SPH high-resolution re-simulation of an individual galaxy halo. The process of setting up the initial conditions is described in detail in Naab et al. (2007) and is briefly reviewed. We have re-run galaxy A at $200^{3}$ particles resolution using a WMAP-1 (Spergel et al. 2003) cosmology with a Hubble parameter of $h=0.65$ ( $\left.\equiv H_{0}=100 h \mathrm{kms}^{-1} \mathrm{Mpc}^{-1}\right)$ with $\sigma_{8}=0.86, f_{b}=\Omega_{b} / \Omega_{m}=0.2, \Omega_{0}=0.3$, and $\Lambda_{0}=0.7$. To re-simulate the target halo at high resolution we increased the particle number to $1.6 \times 10^{7}$ gas and dark matter particles within a cubic volume at redshift $z=24$ containing all particles that end up within the virialized region (we assumed a fixed radius of $0.5 \mathrm{Mpc}$ ) of the halo 
at $z=0$. The tidal forces from particles outside the high resolution cube were approximated by increasingly massive dark matter particles in 5 nested layers of decreasing resolution. The galaxy was not contaminated by massive boundary particles within the virial radius.

The simulation was performed with GADGET-2 (Springel 2005) on Woodhen at the Princeton PICSciE HPC center using a total of 177,000 CPU hours on 64 CPUs. We used a fixed comoving softening until $z=9$, and thereafter the softening, e.g. for the stars, remained fixed at physical $125 \mathrm{pc}$. The mass of an individual stellar particle is $1.3 \times 10^{5} M_{\odot}$ and we spawn two stars per SPH particle.

Star formation and feedback from supernovae was included using the sub-grid multiphase model of (Springel \& Hernquist 2003). We require an over-density contrast of $\Delta>55.7$ for the onset of star formation to avoid spurious star formation at high redshift. The threshold number density for star formation is $n_{\text {thresh }}=0.205 \mathrm{~cm}^{-3}$ and the star formation time-scale is $t_{*}=1.5 h^{-1}$ Gyrs. We also included an uniform UV background radiation field peaking at at $z \simeq 2-3$ (see Naab et al. 2007).

At present the galaxy has a total virial mass of $1.9 \times 10^{12} M_{\odot}$ and a total stellar mass of $1.5 \times 10^{11} M_{\odot}$. The ratio of central stellar mass to halo mass is about a factor of two larger than predicted from gravitational lensing studies (Mandelbaum et al. 2006), however, it is comparable to some recent predictions derived for the Milky Way halo ( e.g. Xue et al. 2008, see however Li \& White 2008). The central stellar component resembles an elliptical galaxy with properties very similar to the results presented in Naab et al. (2007) and in this letter we only focus on particular aspects of the assembly and size evolution of the stellar component.

\section{Results}

During the assembly of the central galaxy we have separated the stars within a fiducial radius of $30 \mathrm{kpc}$ in fixed physical coordinates into stars that have formed in-situ from gas within the galaxy and stars that have formed outside this radius and were accreted later-on. The mass assembly history of the in-situ and accreted components of the stellar system are shown in Fig. 1. The early mass evolution at $z>2$ is driven by the assembly of in-situ stars with a decreasing contribution towards $z \approx 0.7$. Below this redshift only few stars are formed within $30 \mathrm{kpc}$. The final $20 \%$ of stars are added thereafter by accretion of systems formed outside the main stellar system at radii larger than $30 \mathrm{kpc}$.

The upper panel of Fig. 2 shows the density profiles of the in-situ stars at redshifts $\mathrm{z}=5,3,2,1,0$. Between $\mathrm{z}=5$ and $\mathrm{z}=3$ the central galaxy is still building up from gas flows 
feeding the central region of the galaxy directly, forming a concentrated stellar system. The in-situ central stellar densities decrease by more than an order of magnitude towards lower redshifts. The spherical half-mass radii of the in-situ stellar component, show that the in-situ system is very compact (see also Joung et al. 2009) at $z=3(0.5-0.6 \mathrm{kpc}$ ) and its size increases by about a factor of four $(\approx 2 \mathrm{kpc})$ until $\mathrm{z}=0$. In the bottom panel of Fig. 2 we show the density profiles for the stars that have formed outside $30 \mathrm{kpc}$ and have been accreted later-on. This component is more extended at all redshifts and has a shallower density profile. Its central density stays almost constant at $\approx 10^{10} M_{\odot} \mathrm{kpc}^{-3}$ while the density at larger radii subsequently increases towards $z=0$. The half-mass radius of this component is significantly larger than for the in-situ stars $(>3 \mathrm{kpc})$. The central part of the galaxy is always dominated by in-situ stars whereas at redshifts below $z \approx 2-3$ and at radii larger than $\approx 2-3 \mathrm{kpc}$ the system is dominated by accreted stars.

In Fig. 3 we show the time evolution of the edge-on projected half mass radius of stars in the central galaxy within the central physical $30 \mathrm{kpc}$ as a function of time. At $z=3$ the stellar system resembles a compact disk-like or bar-like object with a peak ellipticity of $\epsilon=0.65$ and a size of $\approx 0.3-0.7 \mathrm{kpc}$ at $\mathrm{z}=3$. Thereafter its size increases by a factor of $\approx 3-4$ to its present value of $2.7 \mathrm{kpc}$. We also plot the projected half-light radii in the rest frame K- and V-band using the stellar population models of Bruzual \& Charlot (2003) assuming solar metalicity. In general the half-mass radii trace the half-light radii even at larger redshifts reasonably well.

The K-band rest-frame surface brightness profiles for edge-on projections at $\mathrm{z}=0$ and $\mathrm{z}=3$ are shown in Fig. 4 in combination with the best fitting Sersic profiles. using the fitting procedure of Naab \& Trujillo (2006) excluding the central three softening lengths. At high redshift the system is very compact, $r_{e}=0.69 \mathrm{kpc}$, and has a moderate Sersic index of $n \approx 2.3$. This is in agreement with the system being flattened and disk-like. At low redshift the system is more extended $r_{e}=2.4 \mathrm{kpc}$, and its Sersic index has increased to $n=3.3$. The galaxy is slightly more compact than typical SDSS early-type galaxies at this mass but lies within the observed distribution (Shen et al. 2003, see also Franx et al. 2008). The errors given in the figure are bootstrap errors for a fixed projection. As we have shown before the evolution in surface brightness is mainly driven by an evolution in surface density and not by stellar evolution.

At $\mathrm{z}=3$ the system has a total stellar mass of $M=5.5 \times 10^{10} M_{\odot}$ with an effective radius $r_{\mathrm{eff}}=0.69 \mathrm{kpc}$ and a corresponding effective density of $\rho_{\mathrm{eff}}=0.5 \mathrm{M} /\left(4 / 3 \pi r_{\mathrm{eff}}^{3}\right)=$ $1.6 \times 10^{10} M_{\odot}$. The projected stellar line-of-sight velocity dispersion is $\sigma_{\text {eff }} \approx 240 \mathrm{kms}^{-1}$. The corresponding values at $\mathrm{z}=0$ are $M=15 \times 10^{10} M_{\odot}, r_{\text {eff }}=2.4 \mathrm{kpc}, \rho_{\text {eff }}=1.3 \times 10^{9} M_{\odot}$ and $\sigma_{\text {eff }} \approx 190 \mathrm{kms}^{-1}$, which is a typical dispersion for early-type galaxies at this mass 
(Bender et al. 1992). From $\mathrm{z}=3$ to $\mathrm{z}=0$ the system accretes about $5.5 \times 10^{10} M_{\odot}(\operatorname{see}$ Fig 1 ) and we can assume for the above scaling relations $\eta=1$ and $\epsilon<<1$ (which is a reasonable assumption as most mass is accreted in very small systems and we found the most massive merger since $\mathrm{z}=3$ with a mass ratio of $8: 1$ ). The $\mathrm{z}=0$ values of the simulated galaxy are close to the simple prediction, however, the size increase as well as the decrease in density and dispersion are more moderate. This is however expected as the real evolution of the system is more complex and there is non-negligible in-situ star formation between $\mathrm{z}=3$ and $\mathrm{z}=1$. Still, the simple scaling relations for stellar accretion represent the evolution of the system from $\mathrm{z}=3$ to $\mathrm{z}=0$ very well. However, observations of more massive ellipticals than the one presented here indicate an even stronger size increase (Franx et al. 2008). This effect can be expected if the assembly of more massive galaxies is even more dominated by minor mergers and stellar accretion.

In particular, the drop in velocity dispersion is in qualitative agreement with first direct observations by Cenarro \& Trujillo (2009). Recently, Bezanson et al. (2009) have reported a relatively weak evolution of the density within fixed $1 \mathrm{kpc}$ of only a factor $2-3$. This also is in qualitative agreement with our simulation which shows a decrease of only a factor 1.5 from $\mathrm{z}=2$ to $\mathrm{z}=0$. We also note that the stellar population of the system is already evolved at high redshift. At $z=2$ the galaxy has a stellar mass of $7.5 \times 10^{10} M_{\odot}$ and a local star formation rate of only $\approx 6 M_{\odot} \mathrm{yr}^{-1}$ and an average stellar age of 1.6 Gyrs.

\section{Conclusion \& Discussion}

In this paper we show that the observed size and density evolution of massive spheroids agrees with what is to be expected from a high resolution cosmological simulation of a system which grows at late times predominantly by minor mergers and accretion of stars. We can successfully apply simple scaling relations derived from the virial theorem to demonstrate that that the size increase and decrease in density and velocity dispersion is a natural consequence of mass assembly by much less massive stellar systems and accretion (Cenarro \& Trujillo 2009) and cannot be explained by mass assembly histories dominated by major stellar mergers.

In the simulation, a first phase $(6>z>2)$ dominated by in-situ star formation from inflowing cold gas (see e.g. Dekel et al. 2009 and references therein) produces a massive and dense stellar system with sizes $r_{1 / 2} \leq 1 \mathrm{kpc}$. This phase of the formation of the cores of ellipticals is followed by an extended phase $(3>z>0)$ with little in-situ star formation but significant accretion of stellar material. This material can be stripped at larger radii and increases the size of the system with time. At the same time the central concentration 
is reduced by dynamical friction from the surviving cores (see El-Zant et al. 2001). The apparent size increase is caused by the initial dominance of the in-situ component being heated and, at larger radii, overshadowed ultimately by the accreted stars. From $\mathrm{z}=3$ to $\mathrm{z}=0$ the effective radius of the system increases by a factor of 3.5 with a decrease in the effective density of more than an order of magnitude and a decrease in velocity dispersion of $20 \%$, in good agreement with predictions from simple scaling relations for the accretion of minor mergers.

Detailed investigations of dark matter simulations (see e.g. Genel et al. 2008b; Fakhouri \& Ma 2008; Genel et al. 2008a) as well as recent observations (see e.g. Bundy et al. 2009) on the mass assembly mechanisms of early-type galaxies and their dark matter halos demonstrate the significance of minor mergers. In addition, due to the shape of the mass function, massive systems at high redshift are more likely to experience minor mergers, than lower mass galaxies. If the size evolution is in general driven by minor mergers we would expect a differential size increase, e.g. more massive high redshift systems grow larger than lower mass systems. At the same time minor mergers do also play an important role for the gravitational heating of halo gas, thereby suppressing the formation of new stars (Johansson et al. 2009b).

A picture of a two phase formation process for massive spheroidal galaxies has a number of virtues. In the first dissipative phase at high redshift stars form quickly and build the compact progenitor of present-day ellipticals. In fact, it seems of minor importance if the gas is funneled to the center through streams or mergers of extended gas dominated disks (see e.g. Robertson et al. 2006; Johansson et al. 2009a; Dekel et al. 2009) as long as it happens on a short timescale. The stars formed at this early phase are expected to be significantly enriched in alpha-elements as expected from observations (Thomas et al. 2005) and form the compact core of the elliptical galaxy. Later on metal-poor stars from smaller systems are accreted and form the halo of the galaxy resulting in the observed metalicity gradient. This inside out formation scenario is also made plausible by recent observations of Bezanson et al. (2009). There is an important test of the picture presented in this paper. If correct, then the outer parts of massive giant ellipticals will tend to be old, blue, metal-poor and relatively uniform from galaxy to galaxy since they are all composed essentially of the debris from tidally destroyed accreted small systems.

The simulation was performed at the Princeton PICSciE HPC center. This research was supported by the DFG cluster of excellence 'Origin and Structure of the Universe'. We thank Marijn Franx, Pieter van Dokkum and Ignacio Trujillo for helpful comments on the manuscript. 


\section{REFERENCES}

Bell, E. F., Naab, T., McIntosh, D. H., Somerville, R. S., Caldwell, J. A. R., Barden, M., Wolf, C., Rix, H.-W., Beckwith, S. V., Borch, A., Häussler, B., Heymans, C., Jahnke, K., Jogee, S., Koposov, S., Meisenheimer, K., Peng, C. Y., Sanchez, S. F., \& Wisotzki, L. 2006a, ApJ, 640, 241

Bell, E. F., Phleps, S., Somerville, R. S., Wolf, C., Borch, A., \& Meisenheimer, K. 2006b, ApJ, 652, 270

Bender, R., Burstein, D., \& Faber, S. M. 1992, ApJ, 399, 462

Bezanson, R., van Dokkum, P. G., Tal, T., Marchesini, D., Kriek, M., Franx, M., \& Coppi, P. 2009, ApJ, 697, 1290

Binney, J. \& Tremaine, S. 2008, Galactic Dynamics: Second Edition (Galactic Dynamics: Second Edition, by James Binney and Scott Tremaine. ISBN 978-0-691-13026-2 (HB). Published by Princeton University Press, Princeton, NJ USA, 2008.)

Bruzual, G. \& Charlot, S. 2003, MNRAS, 344, 1000

Bundy, K., Fukugita, M., Ellis, R. S., Targett, T. A., Belli, S., \& Kodama, T. 2009, ArXiv: 0902.1188

Cenarro, A. J. \& Trujillo, I. 2009, ApJ, 696, L43

Cimatti, A., Cassata, P., Pozzetti, L., Kurk, J., Mignoli, M., Renzini, A., Daddi, E., Bolzonella, M., Brusa, M., Rodighiero, G., Dickinson, M., Franceschini, A., Zamorani, G., Berta, S., Rosati, P., \& Halliday, C. 2008, A\&A, 482, 21

Ciotti, L., Lanzoni, B., \& Volonteri, M. 2007, ApJ, 658, 65

Daddi, E., Renzini, A., Pirzkal, N., Cimatti, A., Malhotra, S., Stiavelli, M., Xu, C., Pasquali, A., Rhoads, J. E., Brusa, M., di Serego Alighieri, S., Ferguson, H. C., Koekemoer, A. M., Moustakas, L. A., Panagia, N., \& Windhorst, R. A. 2005, ApJ, 626, 680

Damjanov, I., McCarthy, P. J., Abraham, R. G., Glazebrook, K., Yan, H., Mentuch, E., LeBorgne, D., Savaglio, S., Crampton, D., Murowinski, R., Juneau, S., Carlberg, R. G., Jørgensen, I., Roth, K., Chen, H.-W., \& Marzke, R. O. 2009, ApJ, 695, 101

Dekel, A., Sari, R., \& Ceverino, D. 2009, arXiv:0901.2458

El-Zant, A., Shlosman, I., \& Hoffman, Y. 2001, ApJ, 560, 636 
Fakhouri, O. \& Ma, C.-P. 2008, MNRAS, 386, 577

Fan, L., Lapi, A., De Zotti, G., \& Danese, L. 2008, ApJ, 689, L101

Franx, M., van Dokkum, P. G., Schreiber, N. M. F., Wuyts, S., Labbé, I., \& Toft, S. 2008, ApJ, 688, 770

Genel, S., Genzel, R., Bouché, N., Naab, T., \& Sternberg, A. 2008a, arXiv:0812.3154

Genel, S., Genzel, R., Bouché, N., Sternberg, A., Naab, T., Schreiber, N. M. F., Shapiro, K. L., Tacconi, L. J., Lutz, D., Cresci, G., Buschkamp, P., Davies, R. I., \& Hicks, E. K. S. 2008b, ApJ, 688, 789

Hopkins, P. F., Hernquist, L., Cox, T. J., Dutta, S. N., \& Rothberg, B. 2008, ApJ, 679, 156

Hopkins, P. F., Hernquist, L., Cox, T. J., Keres, D., \& Wuyts, S. 2009, ApJ, 691, 1424

Johansson, P. H., Naab, T., \& Burkert, A. 2009a, ApJ, 690, 802

Johansson, P. H., Naab, T., \& Ostriker, J. P. 2009b, ApJ, 697, L38

Joung, M. R., Cen, R., \& Bryan, G. L. 2009, ApJ, 692, L1

Khochfar, S. \& Burkert, A. 2006, A\&A, 445, 403

Khochfar, S. \& Silk, J. 2006, ApJ, 648, L21

-. 2008, arXiv:0809.1734

Li, Y.-S. \& White, S. D. M. 2008, MNRAS, 384, 1459

Longhetti, M., Saracco, P., Severgnini, P., Della Ceca, R., Mannucci, F., Bender, R., Drory, N., Feulner, G., \& Hopp, U. 2007, MNRAS, 374, 614

Mandelbaum, R., Seljak, U., Kauffmann, G., Hirata, C. M., \& Brinkmann, J. 2006, MNRAS, 368,715

McIntosh, D. H., Guo, Y., Hertzberg, J., Katz, N., Mo, H. J., van den Bosch, F. C., \& Yang, X. 2008, MNRAS, 388, 1537

Naab, T. \& Burkert, A. 2003, ApJ, 597, 893

Naab, T., Johansson, P. H., Ostriker, J. P., \& Efstathiou, G. 2007, ApJ, 658, 710

Naab, T., Khochfar, S., \& Burkert, A. 2006, ApJ, 636, L81 
Naab, T. \& Trujillo, I. 2006, MNRAS, 369, 625

Nipoti, C., Londrillo, P., \& Ciotti, L. 2003, MNRAS, 342, 501

Robertson, B., Hernquist, L., Cox, T. J., Di Matteo, T., Hopkins, P. F., Martini, P., \& Springel, V. 2006, ApJ, 641, 90

Ruszkowski, M. \& Springel, V. 2009, arXiv:0902.0373

Saracco, P., Longhetti, M., \& Andreon, S. 2008, ArXiv: 0810.2795

Shen, S., Mo, H. J., White, S. D. M., Blanton, M. R., Kauffmann, G., Voges, W., Brinkmann, J., \& Csabai, I. 2003, MNRAS, 343, 978

Spergel, D. N., Verde, L., Peiris, H. V., Komatsu, E., Nolta, M. R., Bennett, C. L., Halpern, M., Hinshaw, G., Jarosik, N., Kogut, A., Limon, M., Meyer, S. S., Page, L., Tucker, G. S., Weiland, J. L., Wollack, E., \& Wright, E. L. 2003, ApJS, 148, 175

Springel, V. 2005, MNRAS, 364, 1105

Springel, V. \& Hernquist, L. 2003, MNRAS, 339, 289

Thomas, D., Maraston, C., Bender, R., \& de Oliveira, C. M. 2005, ApJ, 621, 673

Toft, S., van Dokkum, P., Franx, M., Labbe, I., Förster Schreiber, N. M., Wuyts, S., Webb, T., Rudnick, G., Zirm, A., Kriek, M., van der Werf, P., Blakeslee, J. P., Illingworth, G., Rix, H.-W., Papovich, C., \& Moorwood, A. 2007, ApJ, 671, 285

Trujillo, I., Conselice, C. J., Bundy, K., Cooper, M. C., Eisenhardt, P., \& Ellis, R. S. 2007, MNRAS, 382, 109

van der Wel, A., Bell, E. F., van den Bosch, F. C., Gallazzi, A., \& Rix, H.-W. 2009, arXiv:0903.4857

van Dokkum, P. G., Franx, M., Kriek, M., Holden, B., Illingworth, G. D., Magee, D., Bouwens, R., Marchesini, D., Quadri, R., Rudnick, G., Taylor, E. N., \& Toft, S. 2008, ApJ, 677, L5

Whitaker, K. E. \& van Dokkum, P. G. 2008, ApJ, 676, L105

Xue, X. X., Rix, H. W., Zhao, G., Re Fiorentin, P., Naab, T., Steinmetz, M., van den Bosch, F. C., Beers, T. C., Lee, Y. S., Bell, E. F., Rockosi, C., Yanny, B., Newberg, H., Wilhelm, R., Kang, X., Smith, M. C., \& Schneider, D. P. 2008, ApJ, 684, 1143 
This preprint was prepared with the AAS IATEX macros v5.2. 


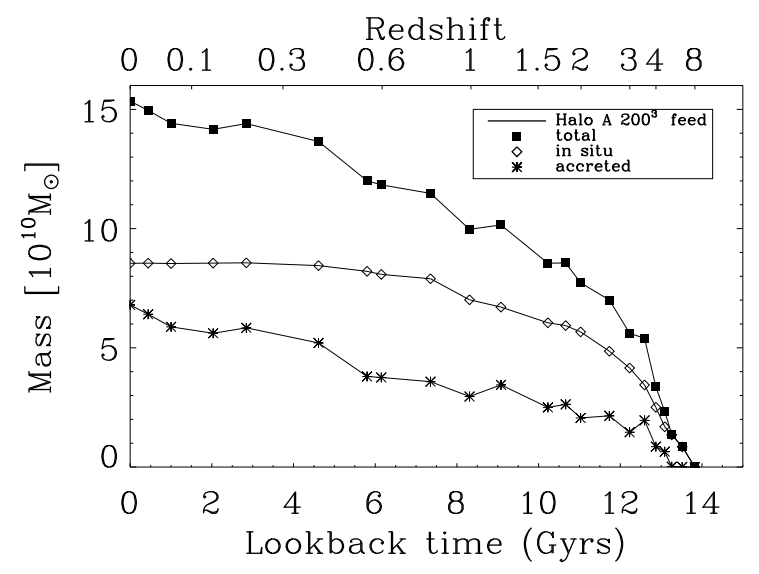

Fig. 1. - Mass assembly history of the stellar system (squares) separated into stars made in-situ (open diamonds) in the galaxy and stars formed outside the galaxy that have been accreted (stars) later on. At high redshift $(z>2)$ the system assembles by the formation of in-situ stars, at low redshift $(z<1)$ accretion is more dominant. 

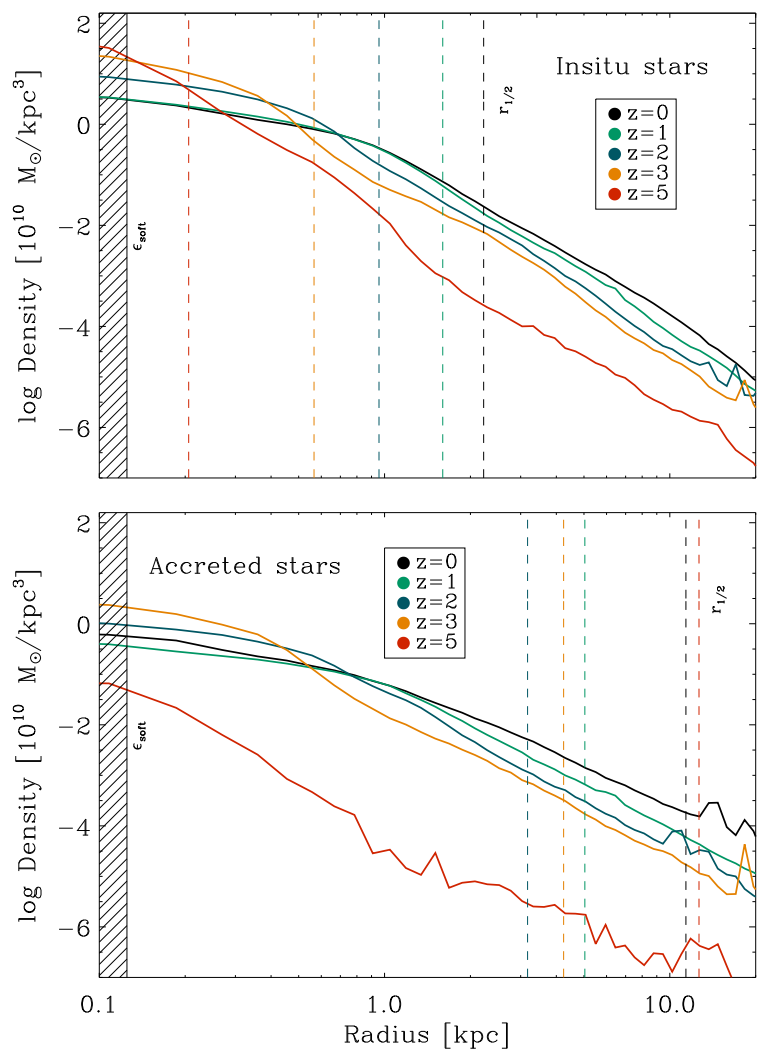

Fig. 2.- Density profile of the stars formed in-situ in the galaxy (upper plot) and of stars formed outside the galaxy and then accreted later-on (bottom plot) at redshifts $\mathrm{z}=5,3,2,1,0$ (red,orange,blue,green,black). The spherical half mass radii $r_{1 / 2}$ are indicated by the dashed vertical lines. The shaded area indicates the gravitational softening length. 


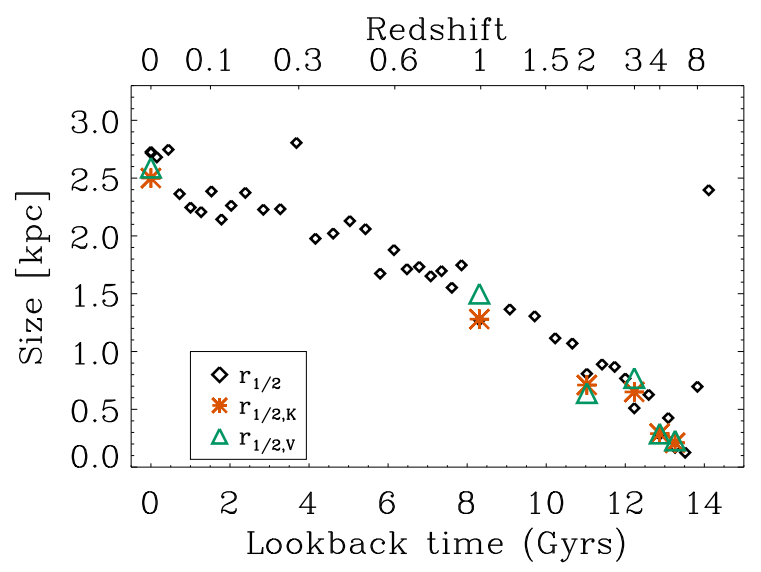

Fig. 3.- Time evolution of the edge-on circular projected stellar half-mass radius within fixed physical $30 \mathrm{kpc}$ (black diamonds). From $z=3-2$ to $z=0$ the size increases by a factor of $\approx 3-4$.We also show the evolution of the rest-frame K-band (red cross) and V-band (green triangle) half-light radius. Outliers indicate minor merger events, e.g. the most massive $(8: 1)$ merger since $\mathrm{z}=3$ at $\mathrm{z}=0.3$.

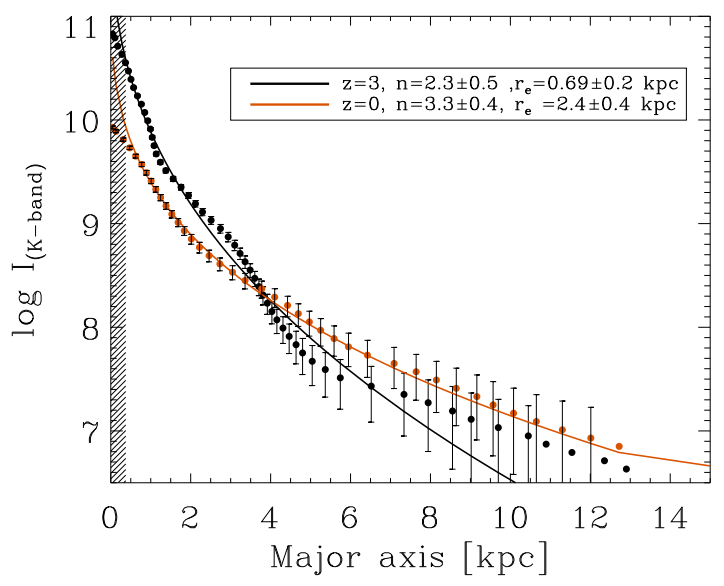

Fig. 4.- K-band surface brightness profiles assuming solar metalicity at $z=3$ (black dots) and $z=0$ (red dots). The black and red lines indicate the best fitting Sersic profile. At high redshift the galaxy has a higher central surface brightness and is more compact. 